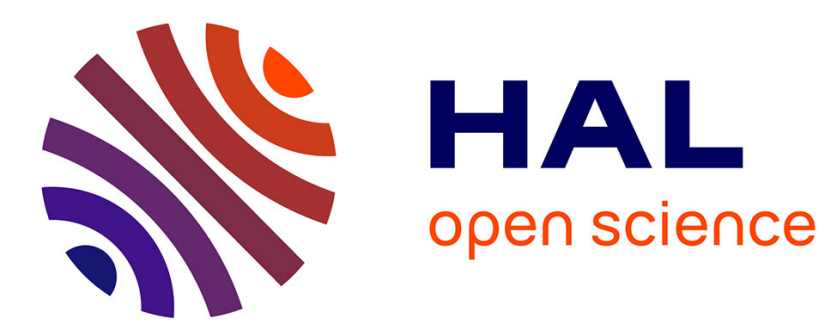

\title{
Simulating wear under cyclic loading by a minimization approach
}

\author{
Michaël Peigney
}

\section{To cite this version:}

Michaël Peigney. Simulating wear under cyclic loading by a minimization approach. International Journal of Solids and Structures, 2004, 41, pp.6783-6799. 10.1016/j.ijsolstr.2004.05.022 . hal00111404

\section{HAL Id: hal-00111404 \\ https://hal.science/hal-00111404}

Submitted on 17 Jul 2019

HAL is a multi-disciplinary open access archive for the deposit and dissemination of scientific research documents, whether they are published or not. The documents may come from teaching and research institutions in France or abroad, or from public or private research centers.
L'archive ouverte pluridisciplinaire HAL, est destinée au dépôt et à la diffusion de documents scientifiques de niveau recherche, publiés ou non, émanant des établissements d'enseignement et de recherche français ou étrangers, des laboratoires publics ou privés. 


\title{
Simulating wear under cyclic loading by a minimization approach
}

\author{
Michaël Peigney \\ Laboratoire de M'ecanique des Solides, Ecole Polytechnique, 91128 Palaiseau cedex, France
}

\begin{abstract}
A new method is developed to determine the asymptotic state reached by a solid continuum subjected to wear and submitted to a cyclic loading. The main idea is to express the stabilized state as the solution of a minimization problem. This approach is applied to the wear problem of a half-plane in contact with a cyclically moving indenter.
\end{abstract}

Keywords: Cyclic loading; Minimization; Wear; Contact mechanics; Green functions

\section{Introduction}

Wear of engineering components can reduce the product life significantly, therefore, its prediction is an important issue in structural design. This supposes to have: (a) a wear model which fully describes the phenomenon, and (b) efficient numerical methods for simulation. A lot of work has been done on (a) (see Meng and Ludema (1995) for an extensive review). This paper addresses the point (b), focusing more precisely on cyclic loadings.

Simulating the evolution of wear is known to lead to substantial numerical difficulties, as pointed out for instance by Põdra and Andersonn (1999). Indeed such problems involve two coupled nonlinearities, namely the wear criterion and the contact conditions. Moreover, the change of geometry caused by wear implies that some remeshing is necessary. These difficulties really come to a head when cyclic loadings are considered, because the simulation over a large number of cycles is often required. This last point is illustrated by the problem pictured in Fig. 1, where the wear problem of a half-plane in contact with a cyclically moving indenter is considered. Fig. 2 shows some experimental results obtained by Fouvry (2001) when the normal force $L$ on the indenter is fixed. If the normal force $L$ is small enough, the wear volume reaches a limit after a large number of cycles: there exists an asymptotic stabilized state. If $L$ is large, the wear volume

\footnotetext{
*Address: Department of Applied Mathematics and Theoretical Physics, Centre for Mathematical Sciences, Wilberforce Road, Cambridge CB3 0WA, UK. Tel.: +44-1223-337887; fax: +44-1223-365900.

E-mail address: michael.peigney@polytechnique.org (M. Peigney).
} 


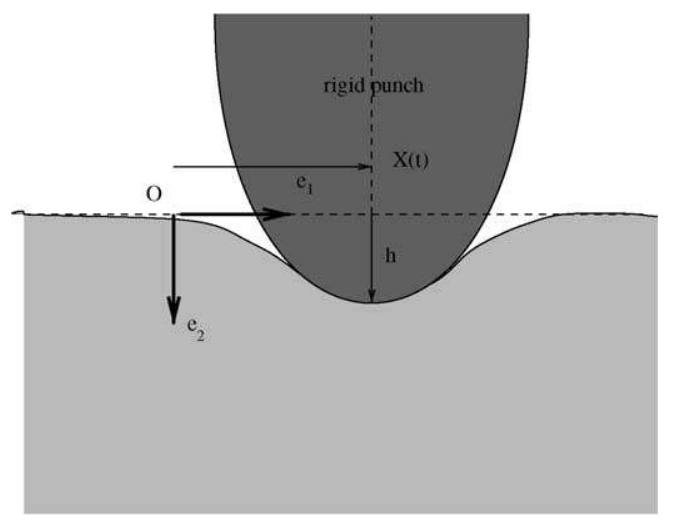

Fig. 1. Wear of a half-plane.

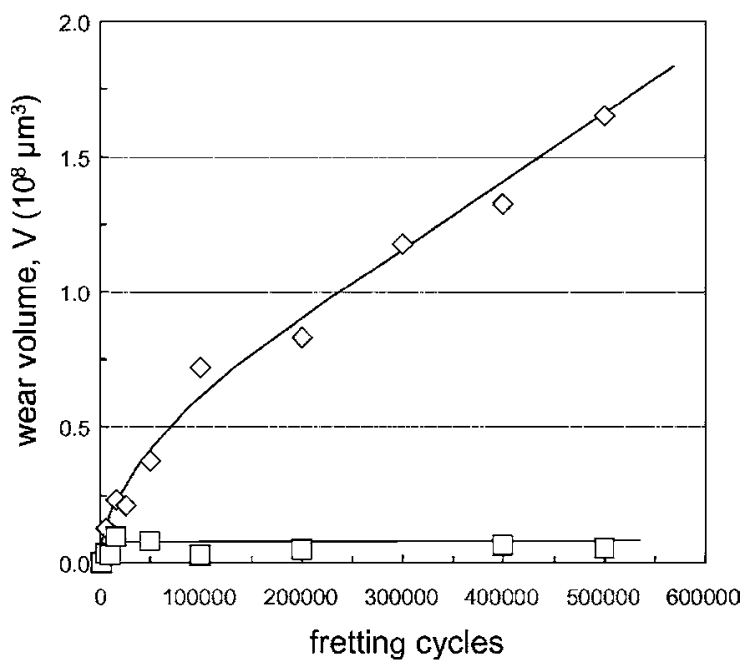

Fig. 2. Wear measures under cyclic loading (from Fouvry, 2001).

is not bounded, but there is still a stabilization in the sense that the loss of volume per loading cycle becomes constant. This last behaviour reminds of the ratchetting of elasto-plastic structures (Koiter, 1960).

One can expect to get a good approximation of this stabilized state by computing the incremental evolution over a sufficiently large number of cycles. The trouble is that this procedure involves prohibitive calculation costs. Indeed the convergence of the incremental evolution towards the asymptotic state can be very slow: as shown in Fig. 2, the asymptotic state is reached after about 50,000 cycles. This difficulty raises the interest of alternative methods which are able to give some information about the stabilized state without computing the complete evolution of wear.

Some contribution in that direction has been provided by Öqvist (2001), using a jump cycle technique. Here we propose a completely different approach, where the main idea is to find the asymptotic state by solving a minimization problem. This approach is used to study a wear problem where displacements are prescribed on the indenter. Therefore, the problem tackled in this paper differs from the experiment displayed in Figs. 1 and 2, where tractions were specified. As will be shown in Section 3, imposing displace- 
ments excludes the possibility of unlimited wear: the geometry of the worn surface necessarily reaches a stabilized profile. Its determination (along with the determination of the stresses in the solids considered) is the central issue.

In Section 2 is introduced a simplified modelling of the wear problem. Although severe assumptions are made, this modelling retains the key ingredients of the mechanical problem, namely the coupling between wear and contact, and the geometry dependence of the Green functions. In this framework, the asymptotic state is estimated by minimizing the energy dissipated in the wear process. The corresponding minimization problem is studied in Sections 3 and 4. Some numerical results are given in Section 5 to illustrate the method.

\section{Modelling of the mechanical problem}

\subsection{Problem considered}

We study the wear problem of an elastic half-plane $\mathscr{P}$ in contact with a rigid indenter (Fig. 1). This problem is considered as 2-dimensional in the plane $\left(\boldsymbol{e}_{1}, \boldsymbol{e}_{2}\right)$ (both strain and stress are plane). The indenter is infinitely long in the third dimension. The indentation depth $h$ being fixed, the indenter undergoes a cyclic translation $X(t) \boldsymbol{e}_{1}$ where $X(t)$ is a $T$-periodic function (i.e., verifying $X(t)=X(t+T)$ ). The function $X$ is supposed to be bounded. Quasi-static evolutions are considered. The constitutive material of $\mathscr{P}$ is supposed linear elastic and homogeneous. We note $\boldsymbol{L}$ the elastic tensor which relates the stress $\sigma$ and the strain $\boldsymbol{\epsilon}$ by

$$
\boldsymbol{\sigma}=\boldsymbol{L}: \boldsymbol{\epsilon}
$$

Because of wear, the profile of $\mathscr{P}$ does not remain flat after the loading is removed. We introduce a positive function $\eta(x, t)$ of abscissa $x$ and time $t$ to describe the profile $\Gamma^{0}(\eta)$ of $\mathscr{P}$ in the unloaded configuration (Fig. $3)$. The volume occupied by $\mathscr{P}$ is denoted $\Omega^{0}(\eta)$. In the loaded configuration, the actual profile $\Gamma(\eta)$ differs from $\Gamma^{0}(\eta)$ by some elastic displacements $\boldsymbol{u}(x, t)=\sum_{i=1}^{2} u_{i}(x, t) \boldsymbol{e}_{i}$. Initially the surface of $\mathscr{P}$ is flat, so $\eta(x, 0)=0$. When the loading is applied, wear can occur, causing $\eta$ to increase with time.

For any mechanical quantity $f$ defined over $\Omega^{0}(\eta)$ (such as the displacement $\boldsymbol{u}$ or the stress $\boldsymbol{\sigma}$ ), we denote by $f(x)$ its value at the point of abscissa $x$ on $\Gamma^{0}(\eta)$, i.e., at the point $x \boldsymbol{e}_{1}+\eta(x) \boldsymbol{e}_{2}$.

\subsection{Contact conditions}

Let $\boldsymbol{T}(x, t)=\sum_{i} T_{i}(x, t) \boldsymbol{e}_{i}$ be the contact traction acting on $\mathscr{P}$ at the point of abscissa $x$ at time $t$. We assume small perturbations, which implies that $u$ and $\nabla u$ are small. We also suppose that $\eta$ and $\partial \eta / \partial x$ are

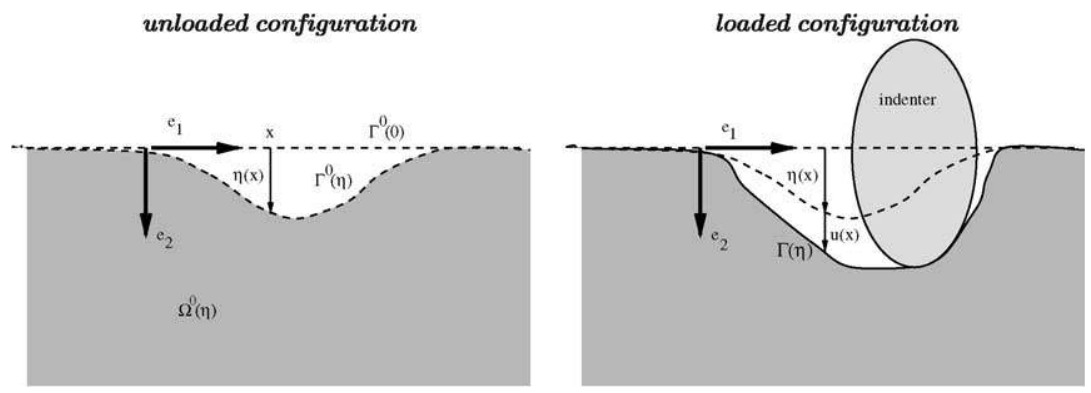

Fig. 3. Tracking of wear. 
small as well. Under these assumptions, contact between the two solids considered is described by the following Signorini conditions:

$$
\begin{aligned}
& T_{2}(x, t) \geqslant 0, v(x, t) \leqslant 0, T_{2}(x, t) \cdot v(x, t)=0 \\
& \text { with } v(x, t)=-u_{2}(x, t)-\eta(x, t)+g(x, t)
\end{aligned}
$$

The gap function $g$ in (2) describes the shape and the movement of the indenter. Here $g$ takes the form:

$$
g(x, t)=g_{0}(x-X(t))
$$

where $g_{0}$ is a function describing the profile of $\mathscr{P}$.

We make the simplifying assumption that $T_{1}$ is equal to the maximum value allowed by the Coulomb's law, thus considering the most damaging scenario with respect to wear. In this framework, the following relationship holds:

$$
T_{1}(x, t)=\operatorname{sgn}(\dot{X}) \mu_{\mathrm{f}} T_{2}(x, t)
$$

where $\mu_{\mathrm{f}}$ is the friction coefficient.

\subsection{Wear criterion}

The indenter is supposed to be perfectly rigid, so that only $\mathscr{P}$ is subjected to wear. We interpret wear as a phase transformation where small rigid particles are created from the original constitutive material of $\mathscr{P}$. This transformation takes place on a moving surface $\Gamma_{w}$ which at time $t$ propagates from $\Gamma(\eta)$ with a normal velocity $\Phi(x)$ (Fig. 4). The wear particles are supposed to be eliminated as soon as they are created.

The thermodynamical analysis of systems with solid phase transformations has been carried out by Pradeilles-Duval and Stolz (1995). The dissipation $\mathscr{D}$ is found to be

$$
\mathscr{D}=\int_{\Gamma(\eta)} \Phi G \mathrm{~d} a
$$

where

$$
G=G_{1}-G_{2}
$$

and

$$
G_{i}=\frac{1}{2} \sigma_{i}: \epsilon_{i}-\boldsymbol{n} \cdot \boldsymbol{\sigma}_{i} \cdot \nabla \boldsymbol{u}_{i} \cdot \boldsymbol{n}
$$

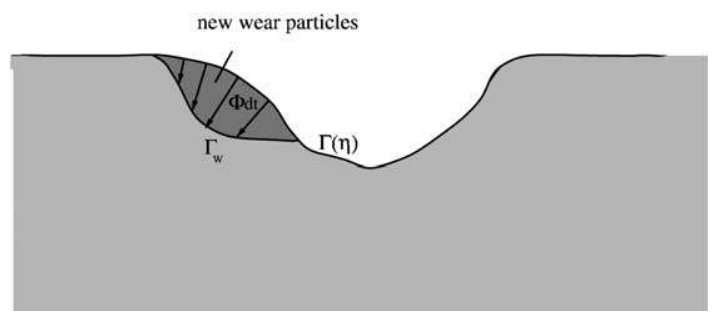

Fig. 4. Formation of wear particles between $t$ and $t+\mathrm{d} t$. 
In (6) and (7), the subscript 1 refers to the domain occupied by the original constitutive material of $\mathscr{P}$, while the subscript 2 refers to the wear particles. The vector $n$ is the normal to $\Gamma(\eta)$. Since the wear particles are supposed to be rigid, we have $\nabla u_{2}=0$ so that $G_{2}$ is null and $G$ is equal to $G_{1}$.

In (5), the energy release rate $G$ appears as the thermodynamical force associated with the normal velocity $\Phi$. Under the assumption of small perturbations $(\boldsymbol{u}, \nabla \boldsymbol{u}, \eta, \partial \eta / \partial x$ small), the dissipation $\mathscr{D}$ can be expressed by

$$
\mathscr{D}=\int_{\Gamma^{0}(0)} \dot{\eta} G \mathrm{~d} x
$$

Following the framework of standard generalized materials (Halphen and Nguyen, 1975), the positivity of $\mathscr{D}$ is ensured if the evolution of $\eta$ is given by

$$
\dot{\eta}=\frac{\mathrm{d} f(G)}{\mathrm{d} G}
$$

where $f$ is a convex positive function such that $f(0)=0$. We here choose the basic form $f=I_{\left.-\infty, G_{c}\right]}$ where $I$ denotes the indicator function and $G_{c}$ is a fixed positive parameter. The evolution law (9) becomes:

$$
\left\{\begin{array}{l}
G<G_{c} \Rightarrow \dot{\eta}=0 \\
G=G_{c} \Rightarrow \dot{\eta} \geqslant 0
\end{array}\right.
$$

Although it may seem rather crude in regard of the numerous wear criterions existing in the literature, the criterion (10) has the advantage of being simple yet thermodynamically consistent. On an interesting note, this thermodynamical approach can be pushed further to get a more realistic wear model, accounting for the influence of a third body in the interface (Dragon-Louiset, 2001). Our primary focus being the simulation strategy, we here restrict ourselves to (10).

\subsection{Geometry dependence of the Green functions}

At any given time $t$, the elastic displacement $\boldsymbol{u}$ and the stress $\boldsymbol{\sigma}$ in $\mathscr{P}$ are solution of the following problem of elasticity:

$$
\begin{array}{ll}
\operatorname{div} \boldsymbol{\sigma}=0 & \text { in } \Omega^{0}(\eta) \\
\boldsymbol{\sigma}(x) \cdot \boldsymbol{n}(x)=\boldsymbol{T}(x) & \text { for all } x \\
\boldsymbol{\sigma}=\boldsymbol{L}: \boldsymbol{\epsilon}(\boldsymbol{u}) & \text { in } \Omega^{0}(\eta) \\
\boldsymbol{\epsilon}(\boldsymbol{u})=\left(\nabla \boldsymbol{u}+{ }^{t} \nabla \boldsymbol{u}\right) / 2 & \text { in } \Omega^{0}(\eta)
\end{array}
$$

To alleviate the notations, the dependence of $\boldsymbol{u}, \boldsymbol{\sigma}, \boldsymbol{T}, \eta$ with respect to time is omitted. The contact conditions and the wear criterion depend on displacements and strains only through their values on the surface. In order to compute the evolution of the system, we thus only need to obtain the values $\boldsymbol{u}(x)$ and $\boldsymbol{\epsilon}(x)$ corresponding to the solution of (11). The displacement $u_{i}(x)$ can be written as

$$
u_{i}(x)=\int_{-b}^{b} \sum_{j=1}^{2} N_{i j}(\eta, x, y) T_{j}(y) \mathrm{d} y
$$

For fixed values of $j$ and $y$, the Green function $N_{i j}(\eta, x, y)$ corresponds to the solution of (11) when $\boldsymbol{T}(x)$ is chosen equal to $\boldsymbol{T}(x)=\delta(x-y) \boldsymbol{e}_{j}$. In (12) the constant $b$ is chosen so that $T_{j}(y)$ is null outside the segment $[-b, b]$.

Note that the strain $\boldsymbol{\epsilon}(x)$ can be deduced from the displacements $\boldsymbol{u}(x)$ on the surface. Indeed derivation of $u_{1}(x)$ with respect to $x$ gives $\epsilon_{11}$. Using the constitutive equation (1) as well as the boundary condition $\boldsymbol{\sigma} \cdot \boldsymbol{n}=\boldsymbol{T}$, one gets the remaining components $\epsilon_{22}$ and $\epsilon_{12}$ of $\boldsymbol{\epsilon}(x)$. 
Eq. (12) shows that calculating the displacement $u_{i}(x)$ is equivalent to computing the Green functions $N_{i j}(\eta, x, y)$ associated with the domain $\Omega^{0}(\eta)$. Since $\eta$ and consequently $\Omega^{0}(\eta)$ evolve as wear occurs, this computation is usually done numerically by remeshing and updating the stiffness matrix. Such operations involve important calculation cost. Using the fact that $\eta$ is small, we avoid this difficulty by deriving some analytical solutions for $N_{i j}(\eta, x, y)$ in the case where $\boldsymbol{L}$ is isotropic. First let us recall that the Green functions $N_{i j}(0, x, y)$ corresponding to $\eta=0$ (half-plane) are well-known for an isotropic linear material with a Young's modulus $E$ and a Poisson's ratio $v$ (see e.g., Johnson (1985)). Substitution in (12) gives the displacement $\boldsymbol{u}^{0}$ solution of (11) when $\eta=0$ :

$$
\begin{aligned}
& u_{1}^{0}(x)=-\frac{(1-2 v)(1+v)}{2 E}\left[\int_{-b}^{x} T_{2}(s) \mathrm{d} s-\int_{x}^{b} T_{2}(s) \mathrm{d} s\right]-\frac{2\left(1-v^{2}\right)}{\pi E} \int_{-b}^{b} T_{1}(s) \ln |x-s| \mathrm{d} s \\
& u_{2}^{0}(x)=-\frac{2\left(1-v^{2}\right)}{\pi E} \int_{-b}^{b} T_{2}(s) \ln |x-s| \mathrm{d} s+\frac{(1-2 v)(1+v)}{2 E}\left[\int_{-b}^{x} T_{1}(s) \mathrm{d} s-\int_{x}^{b} T_{1}(s) \mathrm{d} s\right]
\end{aligned}
$$

Since $\eta$ is small, we wish to use a development of $N_{i j}(\eta, x, y)$ at the first order in $\eta$, i.e.,:

$$
N_{i j}(\eta, x, y)=N_{i j}(0, x, y)+\mathscr{N}_{i j}(x, y) \cdot \eta
$$

where $\mathscr{N}_{i j}(x, y)$ is a linear operator of $\eta$. This development can be performed in closed form, as detailed in Appendix A. For an isotropic material, the corresponding expression of (12) is found to be:

$$
\begin{aligned}
u_{1}(x)= & u_{1}^{0}(x)-\frac{2\left(1-v^{2}\right)}{\pi E} \eta(x) \int_{-b}^{b} \frac{T_{2}(z)}{x-z} \mathrm{~d} z-\frac{(1+v)(1-2 v)}{E} T_{1}(x) \eta(x)+\frac{4\left(1-v^{2}\right)}{\pi E} \int_{-b}^{b} \frac{T_{2}(s) \eta(s)}{x-s} \mathrm{~d} s \\
& +\frac{4\left(1-v^{2}\right)}{\pi^{2} E} \int_{-b}^{b} \frac{\eta(s) F(s)}{s-x} \mathrm{~d} s \\
u_{2}(x)= & u_{2}^{0}(x)-\frac{2\left(1-v^{2}\right)}{\pi E} \int_{-b}^{b} \frac{T_{1}(s) \eta(s)}{x-s} \mathrm{~d} s-\frac{(1+v)(1-2 v)}{E} T_{2}(x) \eta(x)+\frac{4\left(1-v^{2}\right)}{\pi E} \eta(x) \int_{-b}^{b} \frac{T_{1}(s)}{x-s} \mathrm{~d} s
\end{aligned}
$$

with $F(s)=\int_{-b}^{b} \frac{T_{1}(z)}{s-z} \mathrm{~d} z$.

The displacement (15) is the general solution of (11) at the first order in $\eta$.

\section{Formulation of a minimization problem}

The system governed by the equations of Section 2 is a nonlinear system under cyclic loading. The nonlinearities are represented by the contact condition (2) and the wear criterion (10). The cyclic loading is represented by the $T$ - periodic function $g$ which describes the motion of the indenter in (2). The equations derived in Section 2 determine the evolution of the profile $\eta(x, t)$ as time $t$ increases. Here we are interested in the asymptotic behaviour of the system as $t$ tends to infinity.

We define the spatial norm $|\eta(t)|=\max _{x} \eta(x, t)$. Since wear is irreversible, $|\eta(t)|$ is a monotonically increasing function. Therefore, two cases can occur:

(a) if $\max _{t}|\eta(t)|<\infty$ then there exists a function $\eta_{\infty}(x)$ such that $\eta(x, t) \underset{t \rightarrow+\infty}{\rightarrow} \eta_{\infty}(x)$ for all $x$. This case corresponds to limited wear: the geometry of the worn surfaces reaches an asymptotic profile $\eta_{\infty}(x)$ as time tends to infinity;

(b) if $\max _{t}|\eta(t)|=\infty$ then wear is not limited. 
For the problem described in Section 2, displacements are prescribed on the indenter, so wear is necessarily limited. Indeed when $\eta$ is sufficiently large, there is no more contact between the worn half-plane $\mathscr{P}$ and the indenter.

The objective is then to find the stabilized profile $\eta_{\infty}(x)$ and the corresponding stress field in $\mathscr{P}$. An intuitive estimate of the asymptotic wear scar is given by the total area $\mathscr{A}$ swept by the indenter during a loading cycle. The profile $\tilde{\eta}(x)$ of that area $\mathscr{A}$ is defined by $\tilde{\eta}(x)=\max _{t \in[0, T]} g(x, t)$. This estimate is not satisfactory because it completely misses the stress state of the solid during and after the wear: for a scar having the shape $\tilde{\eta}$, the contact tractions are identically null during the loading cycle. Any variation from this shape changes the contact tractions in a nontrivial way, involving the contact conditions (20) and the Green function of the worn half-plane. Therefore, the variation $\eta_{\infty}-\tilde{\eta}$ determines the stress state of the

solid after the wear. Moreover, the contact conditions being highly nonlinear (even in the regularized setting of (20)), the surface tractions depend on the wear depth in a very sensitive way. For these reasons, finding consistent estimates of the stabilized state is not trivial and requires good levels of precision in the computations.

An alternative method to incremental analysis is now introduced for determining the asymptotic state.

The wear criterion (10) implies that the profile $\eta_{\infty}(x)$ must belong to the set $\mathscr{K}$ defined by:

$$
\mathscr{K}=\left\{\eta(x), G(\eta, x, t) \leqslant G_{c} \text { for all }(x, t) \in[-b, b] \times[0, T]\right\}
$$

where $G(\eta, x, t)$ is the energy release rate at abscissa $x$ and time $t$ for the profile $\eta$.

Some additional information is needed to identify $\eta_{\infty}$ within the set of admissible profiles $\mathscr{K}$. Here we propose to approximate $\eta_{\infty}$ by minimizing the total energy dissipated by wear over the time interval $[0,+\infty[$. A similar idea has been proposed by Bui (1994) for determining the equilibrium state of systems with solid phase transformation.

In the problem considered, the energy $\mathscr{W}$ dissipated in the wear process is found by integrating (8) between $t=0$ (when $\eta=0$ ) and $t=+\infty$ (when $\left.\eta=\eta_{\infty}\right)$. We find

$$
\mathscr{W}=G_{c} \int_{-b}^{b} \eta_{\infty}(x) \mathrm{d} x
$$

The asymptotic state is thus estimated by solving the minimization problem

$$
\min _{\eta \geqslant 0, \eta \in \mathscr{K}} J(\eta)
$$

where $J(\eta)=G_{c} \int_{-b}^{b} \eta(x) \mathrm{d} x$.

Since it is not proved that the asymptotic state indeed minimizes the total dissipation, the stabilized state solution of (18) can only be considered as an estimate of the real stabilized state reached by the system. It will be shown in Section 5 that this estimate is physically consistent, in the sense that it agrees qualitatively with experimental results from the literature.

\subsection{Regularization}

In (18), the function $J$ is fairly simple, but the set $\mathscr{K}$ is not. Actually $\mathscr{K}$ is generally not convex and not smooth, because $G(\eta, x, t)$ depends on the contact tractions defined by (2). For the sake of practical considerations the formulation (18) is now modified.

First thing we do is to enforce the admissibility condition $\eta \in \mathscr{K}$ by penalization. That is to say we solve

$$
\min _{\eta \geqslant 0} G_{c} \int_{-b}^{b} \eta(x) \mathrm{d} x+\frac{1}{\alpha} \int_{-b}^{b} \int_{0}^{T}\left\langle G(\eta, x, t)-G_{c}\right\rangle_{+}^{2} \mathrm{~d} x \mathrm{~d} t
$$


where $\alpha$ is a small positive parameter and the positive part $\langle x\rangle_{+}$of a scalar $x$ is defined by $\langle x\rangle_{+}=$ $(x+|x|) / 2$. This formulation removes the difficulty of constructing profiles which a priori verifies the admissibility condition (16).

It has already been noted that $G(\eta, x, t)$ depends on the nondifferentiable contact condition (2). This implies that the function to minimize in (19) is not differentiable as well. Although there do exist some numerical algorithms for solving nonsmooth optimization problems (Tiba, 1990), more complexity and more computation time are involved compared to the differentiable case.

To avoid such complication, we choose to regularize the contact conditions by setting

$$
T_{2}(x)=\gamma(v(x))
$$

with $\gamma$ a convex differentiable function of $x$ such that $\gamma^{\prime}(x) \geqslant 0$. This approach to contact conditions has been developed by (Oden and Martins, 1985) with $\gamma(x)=C_{n}\langle x\rangle_{+}^{m_{n}}$.

Let us now define the differentiable function $J_{\alpha}(\eta)$ :

$$
J_{\alpha}(\eta)=G_{c} \int_{-b}^{b} \eta(x) \mathrm{d} x+\frac{1}{\alpha} \int_{-b}^{b} \int_{0}^{T}\left\langle G(\eta, x, t)-G_{c}\right\rangle_{+}^{2} \mathrm{~d} x \mathrm{~d} t
$$

where $G(\eta, x, t)=\boldsymbol{\sigma}: \boldsymbol{\epsilon} / 2-\boldsymbol{n} \cdot \boldsymbol{\sigma} \cdot \nabla \boldsymbol{u} \cdot \boldsymbol{n}$ and $(\boldsymbol{\sigma}, \boldsymbol{u})$ is the solution of (4), (11) and (20). We are led to solve:

$$
\min _{\eta \geqslant 0} J_{\alpha}(\eta)
$$

where the function $J_{\alpha}$ is differentiable and the set $\eta \geqslant 0$ is convex.

A lot of numerical algorithms could be used to solve such a problem (Fletcher, 1987). Since the function $J_{\alpha}$ is differentiable, an algorithm making use of the gradient $J_{\alpha}^{\prime}$ is a good choice. Such algorithms typically require new computations of $J_{\alpha}^{\prime}$ at each iteration, so the calculation of this gradient is a crucial step in terms of computational costs. The next section focuses on the evaluation of $J_{\alpha}^{\prime}$.

\subsection{Differentiation of $J_{\alpha}$ with respect to the domain $\eta$}

In order to get the expression of $J_{\alpha}^{\prime}(\eta)$, we write down the variation $\delta J_{\alpha}(\eta)$ corresponding to a variation $\delta \eta$ of $\eta$, knowing that

$$
\delta J_{\alpha}(\eta)=\left\langle J_{\alpha}^{\prime}, \delta \eta\right\rangle
$$

where $\langle$,$\rangle denoted the duality bracket. Using (21), we find:$

$$
\delta J_{\alpha}(\eta)=G_{c} \int_{-b}^{b} \delta \eta(x) \mathrm{d} x+\frac{2}{\alpha} \int_{-b}^{b} \int_{0}^{T}\left\langle G(\eta, x, t)-G_{c}\right\rangle_{+} \delta G \mathrm{~d} x \mathrm{~d} t
$$

with

$$
\delta G=\boldsymbol{\epsilon}(\boldsymbol{u}): \boldsymbol{L}: \boldsymbol{\epsilon}(\delta \boldsymbol{u})-\boldsymbol{n} \cdot(\boldsymbol{L}: \boldsymbol{\epsilon}(\delta \boldsymbol{u})) \cdot \nabla \boldsymbol{u} \cdot \boldsymbol{n}-\boldsymbol{n} \cdot(\boldsymbol{L}: \boldsymbol{\epsilon}(\boldsymbol{u})) \cdot \nabla(\delta \boldsymbol{u}) \cdot \boldsymbol{n}
$$

The variation of the displacement $\delta \boldsymbol{u}$ is obtained by differentiating (12):

$$
\delta u_{i}(x)=\int_{-b}^{b} \sum_{j=1}^{2}\left(\delta N_{i j}(\eta, x, y) \cdot T_{j}(y)+N_{i j}(\eta, x, y) \cdot \delta T_{j}(y)\right) \mathrm{d} y
$$

where the time dependence of $\delta u_{i}$ and $T_{j}$ has been omitted. At the first order in $\eta$, the function $N_{i j}(\eta, x, y)$ is given by (14) and the term $\delta N_{i j}(\eta, x, y)$ is equal to $\mathscr{N}_{i j}(x, y) \cdot \delta \eta$. The contact conditions (4) and (20) give:

$$
\begin{aligned}
& \delta T_{1}(x)=\operatorname{sgn}(\dot{X}) \mu_{f} \delta T_{2}(x) \\
& \delta T_{2}(x)=-\gamma^{\prime}(v(x)) \cdot\left(\delta \eta(x)+\delta u_{2}(x)\right)
\end{aligned}
$$


Substituting (26) in (27) provides the following integral equation on $\delta T_{2}$ :

$$
\delta T_{2}(x)+\int_{-b}^{b} f(x, y) \cdot \delta T_{2}(y) d y=g[\delta \eta](x)
$$

with

$$
\begin{aligned}
& f(x, y)=\gamma^{\prime}(v(x))\left(\mathscr{N}_{22}(\eta, x, y)+\mu_{\mathrm{f}} \operatorname{sgn}(\dot{X}) \mathscr{N}_{21}(\eta, x, y)\right) \\
& g[\delta \eta](x)=-\gamma^{\prime}(v(x)) \cdot \delta \eta(x)-\gamma^{\prime}(v(x)) \cdot \int_{-b}^{b} \gamma(v(y))\left(\mathscr{N}_{22}(x, y)+\mu_{\mathrm{f}} \operatorname{sgn}(\dot{X}) \mathscr{N}_{21}(x, y)\right) \cdot \delta \eta \mathrm{d} y
\end{aligned}
$$

We observe that the operator $\delta \eta \rightarrow g[\delta \eta]$ is linear. Let $f^{*}(x, z)$ be the function such that for any fixed value of $z$, the function $x \mapsto f^{*}(x, z)$ is the solution of

$$
f^{*}(x, z)+\int_{-b}^{b} f(x, y) f^{*}(y, z) \mathrm{d} y=-f(x, z)
$$

It is then easy to check that the solution of (28) is

$$
\delta T_{2}(x)=g[\delta \eta](x)+\int_{-b}^{b} f^{*}(x, y) g[\delta \eta](y) \mathrm{d} y
$$

This relationship gives $\delta T_{2}(x)$ as a function of $\delta \eta$. By substituting (31) in (26) and (24), we get $\delta J_{\alpha}$ as a linear form in $\delta \eta$, which corresponds to the gradient $J_{\alpha}^{\prime}$. Unfortunately, Eq. (30) can only be solved numerically, but it still provides an efficient way to calculate $J_{\alpha}^{\prime}$. This is explained in the next section which is devoted to the implementation of the approach presented.

\section{Considerations about the discretized problem}

In practice a discretization both in space and time needs to be performed: the function $\eta(x)$ is represented by a vector $\boldsymbol{p}$ in $\mathbb{R}^{k}$, and the condition (16) is written only at a finite sequence of times $\left\{t_{i}\right\}_{1 \leqslant i \leqslant m}$ in $[0, T]$. In that framework, the problems (18) and (22) become, respectively,

$$
\begin{aligned}
& \left.\min _{\left\{\boldsymbol{p} \geqslant 0 ; \boldsymbol{G}_{i}(\boldsymbol{p}) \leqslant G_{c} \boldsymbol{I}\right.} \text { for } 1 \leqslant i \leqslant m\right\} \\
& \min _{\boldsymbol{p} \geqslant \mathbf{0}} J_{\alpha}^{h}(\boldsymbol{p})
\end{aligned}
$$

where $J^{h}$ and $J_{\alpha}^{h}$ are discretized versions of the functionals defined in (18) and (21), respectively. The functions $J^{h}$ and $J_{\alpha}^{h}$ take the form

$$
\begin{aligned}
& J^{h}(\boldsymbol{p})=|\boldsymbol{p}| \\
& J_{\alpha}^{h}(\boldsymbol{p})=|\boldsymbol{p}|+\frac{1}{\alpha} \sum_{i=1}^{m}\left\langle\boldsymbol{G}_{i}(\boldsymbol{p})-G_{c} \boldsymbol{I}\right\rangle_{+} \cdot \Delta_{1} \cdot\left\langle\boldsymbol{G}_{i}(\boldsymbol{p})-G_{c} \boldsymbol{I}\right\rangle_{+}
\end{aligned}
$$

where || is a norm on $\mathbb{R}^{k}$ and $\Delta_{1}$ is a positive definite matrix. The vector $\boldsymbol{G}_{i}(\boldsymbol{p})$ gives the values of the energy release rate at time $t_{i}$ in the discretized model. The vector $\boldsymbol{I}$ is the vector of same size than $\boldsymbol{G}_{i}$ which all its components equal to 1 . For a fixed time step $t_{i}$, calculating $\boldsymbol{G}_{i}(\boldsymbol{p})$ requires the resolution of the nonlinear system (2), (12) and (20). Therefore, the evaluation of $J_{\alpha}^{h}(\boldsymbol{p})$ involves the resolution of $m$ nonlinear systems.

Since the constraint $\boldsymbol{G}_{i}(\boldsymbol{p}) \leqslant G_{c} \boldsymbol{I}$ is not convex, the existence of solutions to (32) and (33) should be justified. Another theoretical question is the convergence of (33) towards (32) as the penalization parameter $\alpha$ tends to 0 . Both these issues are addressed in Appendix B. 
From a practical point of view, it has already been mentioned that the evaluation of the gradient $J_{\alpha}^{h^{\prime}}(\boldsymbol{p})$ is a crucial stage in terms of computational costs. The most natural way of estimating $J_{\alpha}^{h^{\prime}}(\boldsymbol{p})$ is to compute all the partial derivatives $\partial J_{\alpha}^{h} / \partial p_{i}$ using a finite difference scheme:

$$
\frac{\partial J_{\alpha}^{h}}{\partial p_{i}} \simeq \frac{1}{\theta}\left(J_{\alpha}^{h}\left(p_{1}, \cdots, p_{i}+\theta, \cdots, p_{N}\right)-J_{\alpha}^{h}\left(p_{1}, \cdots, p_{i}, \cdots, p_{N}\right)\right)
$$

where $\theta$ is a fixed parameter. Since each evaluation of $J_{\alpha}^{h}$ requires the resolution of $m$ nonlinear systems, this way of estimating $J_{\alpha}^{h^{\prime}}$ is quite time consuming. Moreover the choice of $\theta$ in (35) can be tricky: $\theta$ should be small enough to get a close approximation of the derivative but large enough to avoid numerical accuracy problems. Part of these difficulties is removed by using the developments of Section 3.2. Indeed it has been showed in Section 3.2 that the gradient $J_{\alpha}^{h^{\prime}}$ can be expressed in terms of the solution of (30). In the discretized model, the integral equation (30) is represented by a linear system which can be solved numerically for each time step. The gradient $J_{\alpha}^{h^{\prime}}$ is thus obtained by solving $m$ linear systems, instead of $k m$ nonlinear systems with the finite difference scheme (35). The difficulty of choosing a good value of $\theta$ in (35) is also removed by using the formulae of Section 3.2.

\section{Application}

In this section we give some numerical results obtained by the proposed method. All the lengths are expressed in a non-dimensional way as multiples of a characteristic length denoted $a$. We choose the function $X(t)$ as $X(t)=a \sin 2 \pi t$. The function $g_{0}$ defining the shape of the indenter is taken as

$$
g_{0}(x)=h-\frac{x^{2}}{2 R}
$$

This equation describes an indenter with radius of curvature $R$. The parameter $h$ is the indentation depth. Expressing all lengths as multiple of $a$, (36) can be rewritten as

$$
\frac{g_{0}}{a}(x / a)=\frac{h}{a}-\frac{1}{2} \frac{a}{R}\left(\frac{x}{a}\right)^{2}
$$

All the results presented in this section correspond to $R / a=10$ and $h / a=0.01$. The constitutive material of the half-plane is taken isotropic with a Young's modulus $E$ equal to $200 \mathrm{MPa}$ and a Poisson's ratio $v$ equal to 0.3 . The constitutive parameter $G_{c}$ is chosen equal to $10^{-4} \mathrm{~J}$.

The surface $\Gamma_{0}(0)$ is discretized using 160 points spaced at equal distances on the interval $-4 \leqslant x_{1} / a \leqslant 4$. The loading cycle is divided in 20 equal time steps. The asymptotic state is then found by solving (33) with a BFGS algorithm (Fletcher, 1987), the penalization parameter $\alpha$ being set equal to $10^{-6}$.

Fig. 5 shows the solution $\eta_{\infty}$ obtained. The curved shape of the asymptotic wear scar is consistent with the experimental results of (Fouvry, 2001) and the numerical simulations of Öqvist (2001). This shows that the strategy of minimizing the total dissipation yields realistic results concerning the asymptotic state of the system.

Figs. 6-9 are related to the stabilized cycle, in which $\eta(x, t)=\eta_{\infty}(x)$ for $t \in[0, T]$. The asymptotic profiles both in the unloaded configuration (dashed line) and in the loaded configuration (solid line) are plotted in the lower half of Figs. 6-9. The position of the indenter at each time considered is also represented by the parabolic profile in dashed line. A little arrow indicates the direction of sliding. On these Figures the vertical displacements are magnified for a better clarity: actually the wear depth is significantly less than the radius of curvature of the indenter. We can observe that the elastic displacements are relatively low compared to the wear depth. This stems from the value chosen for $G_{c}$ : with a bigger value of $G_{c}$, larger 


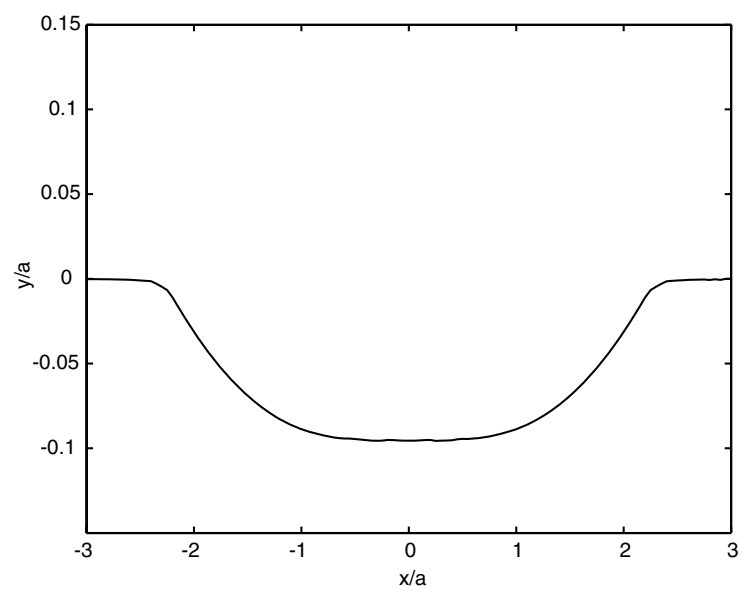

Fig. 5. Asymptotic profile $\eta_{\infty}(x)$.

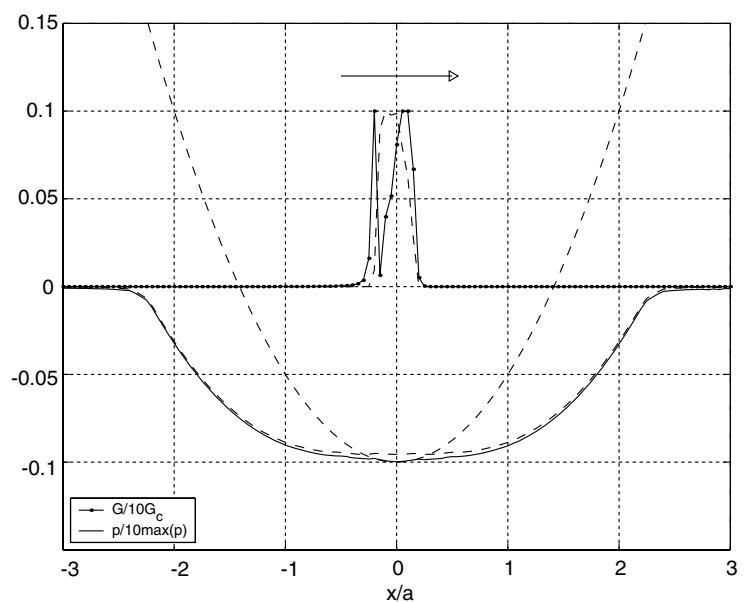

Fig. 6. Evolution on the stabilized cycle at $t / T=0$.

displacements would have been supported by the half-plane and consequently the wear depth would be decreased.

The normalized distributions of the energy release rate $G$ and the contact pressure $p=T_{2}(x)$ are plotted in the upper half of Figs. 6-9. This enables to check that the condition $G\left(\eta_{\infty}, x, t\right) \leqslant G_{c}$ is satisfied all over $\Gamma_{0}\left(\eta_{\infty}\right)$. A gap can be observed in the distribution of $G$, for instance on Fig. 6. Actually this gap is correlated to the distribution of $\epsilon_{11}$, as illustrated in Fig. 12. The curves in Fig. 12 show that the front of the contact area is in compression $\left(\epsilon_{11}<0\right)$ while the back is in traction $\left(\epsilon_{11}>0\right)$. This phenomenon has been observed experimentally by (Barquin et al., 1974). Consequently there is a zero-crossing in the distribution of $\epsilon_{11}$, which corresponds to a decrease of $G$ (indeed $G$ depends on $\epsilon_{11}^{2}$ ).

Fig. 10 shows the influence of wear on the contact tractions. We compare the distribution of tractions at the beginning of a cycle for the initial flat profile $\eta=0$ (distribution $\mathrm{B}$, dashed line) and the asymptotic 


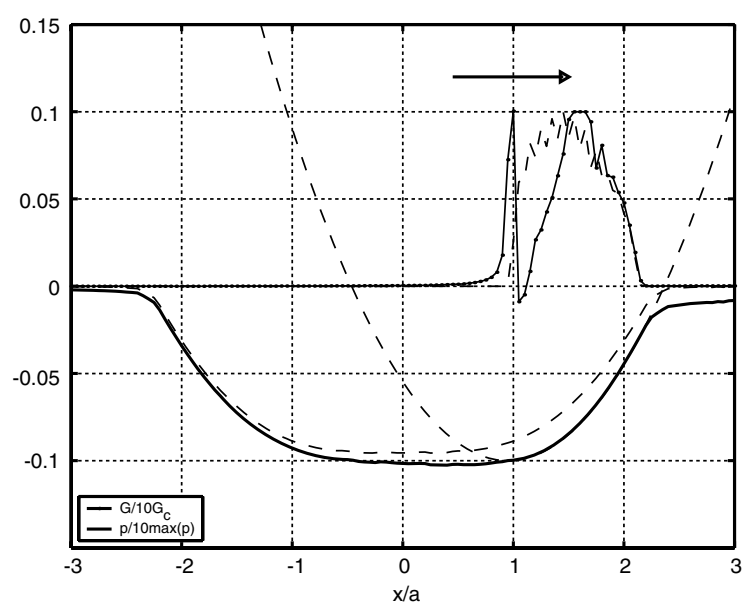

Fig. 7. Evolution on the stabilized cycle at $t / T=0.2$.

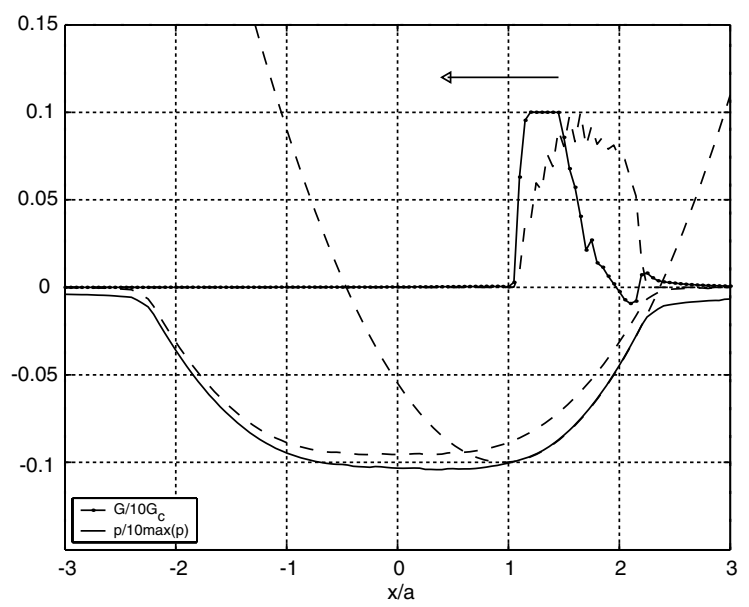

Fig. 8. Evolution on the stabilized cycle at $t / T=0.3$.

worn profile $\eta_{\infty}$ (distribution A, solid line). The contact tractions for $\eta_{\infty}$ are significantly lower, which stems from the fact that displacements are prescribed on the indenter. Indeed the equality $u_{2}(x)+\eta(x)=g(x, t)$ is verified on the contact area. After wear has occurred $(\eta(x)>0)$, the elastic displacement $u_{2}(x)$ is thus decreased. The half-plane is globally less loaded, which results in a decreased distribution of tractions on the surface.

On Fig. 10 is also plotted the distribution of contact tractions for the initial plane profile, in the frictionless case (distribution $\mathrm{C}$ ). The symmetry axis of this distribution corresponds to the symmetry axis of the indenter. This enables to observe that the distributions A and B are shifted in the direction opposite of sliding. This is a consequence of friction, which has already been exhibited by (Bui and Dang Van, 1976) in the problem of a rigid indenter sliding on an elastic half-plane. At $t / T \simeq 0.25$ on the stabilized cycle, we 


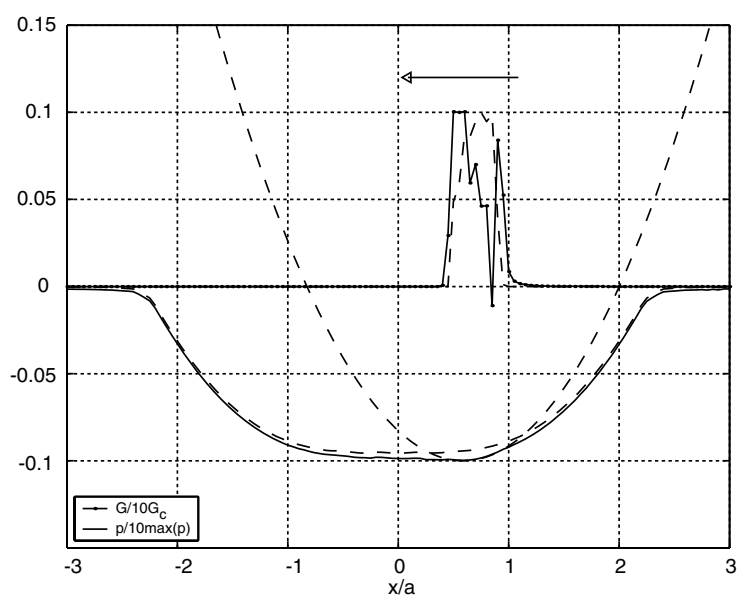

Fig. 9. Evolution on the stabilized cycle at $t / T=0.4$.

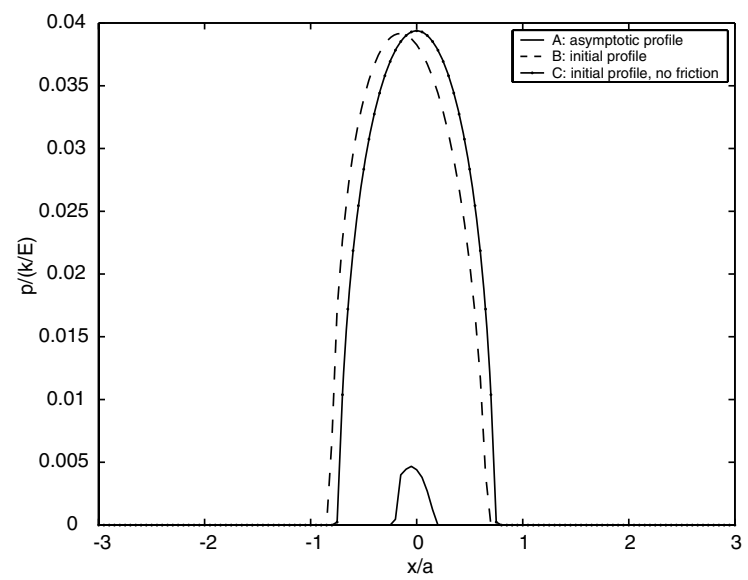

Fig. 10. Contact pressures at the beginning of the stabilized cycle.

observe that the distribution of tractions is now shifted in the direction of the sliding (Fig. 11): here the contact area is determined by the shape of the worn scar.

The solution $\eta_{\infty}$ is obtained after about 1000 iterations of the minimization algorithm. For each iteration, the most time consuming steps are the computation of $J_{\alpha}^{h}$ (resolution of $m$ nonlinear systems) and $J_{\alpha}^{h^{\prime}}$ (resolution of $m$ linear systems). Integrating the evolution of the system over a cycle would typically require the resolution of $m$ nonlinear systems, so the computational cost of the minimization procedure is roughly that of 2000 loading cycles calculated with a step-by-step algorithm. Wear under cyclic loading is known to be a slow process: for instance, in the experiment presented in Section 1, the stabilization occurs after about 50,000 cycles. This suggests that an incremental procedure, which traces the complete evolution of the structure, would require the computation of a very large number of cycles in order to reach the stabilization. Therefore, there should be a substantial gain in using the approach presented. 


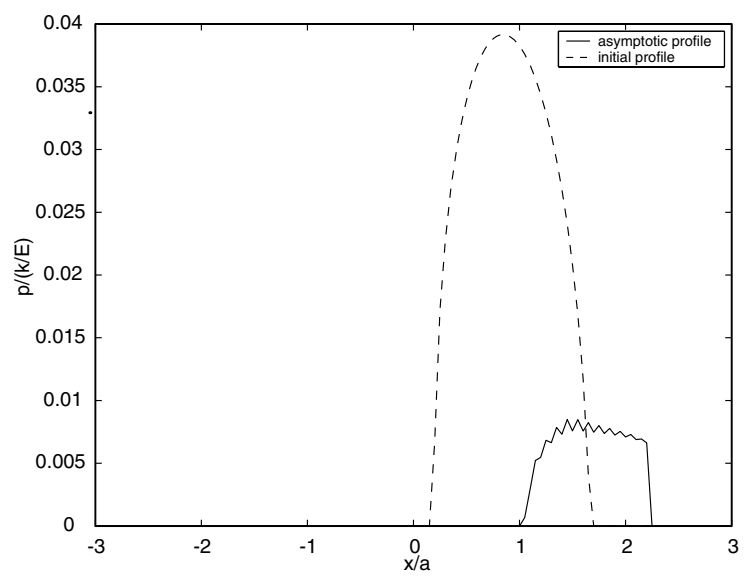

Fig. 11. Stabilized contact pressures at $t / T=0.25$.

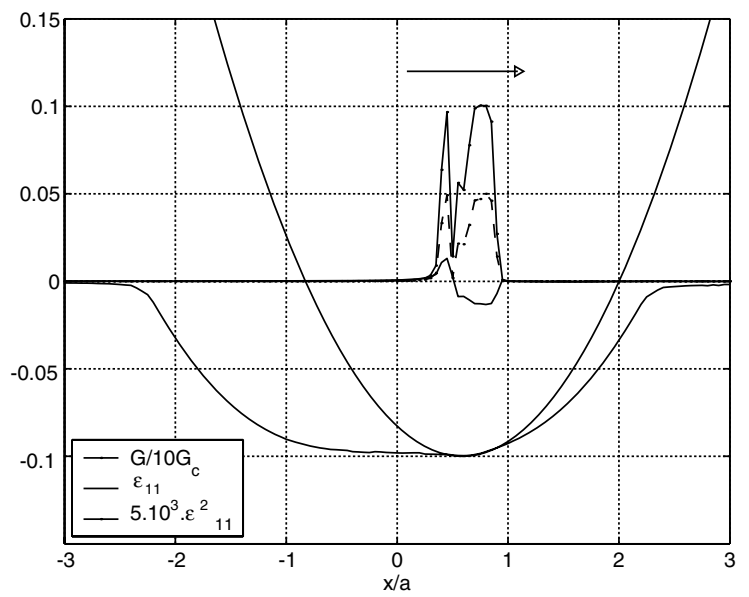

Fig. 12. Strain component $\epsilon_{11}$ at $t / T=0.1$.

\section{Conclusion}

In this paper, a minimization approach has been introduced to estimate the asymptotic state for wear problems under cyclic loadings. Even though the mechanical problem has been simplified considerably, the results obtained remain consistent with experimental measures and numerical simulations from the literature. The computational costs needed to get the asymptotic state are expected to be reduced significantly by comparison with the incremental procedure. The implementation of the method presented is easy, as numerical algorithms for solving such minimization problems are readily available in standard computing software.

In view of these first results, this minimization approach is worth being developed further. Even if this method is approximative in essence, the simplicity of its implementation and its expected efficiency could make it useful in the early stage of structural design. Moreover, some of the ideas and techniques used here 
(like the Green functions for the worn-out half plane) could be of some interest for other problems related to wear research.

Possible developments of the approach presented include most notably taking stick/slip areas into account, and considering a more realistic wear model. As mentioned in Section 2, an interesting choice is the wear model of Dragon-Louiset (2001), which generalizes the model used in this paper. It should also be emphasized that the method presented is not restricted to the half-plane and could be applied on other plane problems. However, in that case the Green functions should be evaluated numerically.

The formulation of the asymptotic state as the solution of a minimization problem bears some promising potentialities. For example, if some a priori information is known about the shape of the asymptotic wear scar, then the optimization procedure could be restricted to the set of profiles which are compatible with this information. This way the asymptotic state should be reached more rapidly. In the long run, the method described here could be combined with a minimization approach developed for elasto-plastic structures under cyclic loadings (Peigney and Stolz, 2003). Putting these two approaches together into an unified minimization problem would allow to tackle the coupling between wear and plasticity in cyclically loaded structures.

\section{Appendix A. Green functions of the worn-out half plane}

For any given $\boldsymbol{x}^{\prime}=x_{1}^{\prime} \boldsymbol{e}_{1}+x_{2}^{\prime} \boldsymbol{e}_{2}$ in $\Omega^{0}(\eta)$ and $j$ in $\{1,2\}$, we consider the displacement $\boldsymbol{x} \mapsto \boldsymbol{N}_{j}\left(\boldsymbol{x}, \boldsymbol{x}^{\prime}\right)$ which is the plane strain solution of the following problem:

$$
\begin{array}{ll}
\boldsymbol{\sigma}_{j}=\boldsymbol{L}: \boldsymbol{\epsilon}\left(\boldsymbol{N}_{j}\right) & \text { in } \Omega^{0}(\eta) \\
\operatorname{div} \boldsymbol{\sigma}_{j}+\delta\left(\boldsymbol{x}-\boldsymbol{x}^{\prime}\right) \cdot \boldsymbol{e}_{j}=0 & \text { in } \Omega^{0}(\eta) \\
\boldsymbol{\sigma}_{j} \cdot \boldsymbol{n}=0 & \text { on } \Gamma^{0}(\eta) \\
\boldsymbol{\epsilon}\left(\boldsymbol{N}_{j}\right)=\left(\nabla_{x} \boldsymbol{N}_{j}+{ }^{t} \nabla_{x} \boldsymbol{N}_{j}\right) / 2 & \text { in } \Omega^{0}(\eta)
\end{array}
$$

The function $\boldsymbol{N}_{j}^{0}\left(\boldsymbol{x}, \boldsymbol{x}^{\prime}\right)$ is defined as the displacement solution of (A.1) when $\eta$ is chosen equal to 0 . For $\boldsymbol{L}$ corresponding to an isotropic behaviour, the expression of $N^{0}$ is well-known. The problem addressed here is to obtain the expression of $\boldsymbol{N}_{j}\left(\boldsymbol{x}, \boldsymbol{x}^{\prime}\right)$ at the first order in $\eta$. This can be achieved using the general formula proved by Kirchner and Ni (1993):

$$
\begin{aligned}
\left(\boldsymbol{N}_{j}(\boldsymbol{X}, \boldsymbol{Y})-\boldsymbol{N}_{j}^{0}(\boldsymbol{X}, \boldsymbol{Y})\right) \cdot \boldsymbol{e}_{i}= & \int_{\Omega^{0}(0)-\Omega^{0}(\eta)} \boldsymbol{\epsilon}\left(\boldsymbol{N}_{j}^{0}\right)(\boldsymbol{x}, \boldsymbol{Y}): \boldsymbol{L}: \boldsymbol{\epsilon}\left(\boldsymbol{N}_{i}^{0}\right)(\boldsymbol{x}, \boldsymbol{X}) \mathrm{d} S \\
& -\int_{\Gamma^{0}(\eta)}\left(\boldsymbol{N}_{i}(\boldsymbol{x}, \boldsymbol{X})-\boldsymbol{N}_{i}^{0}(\boldsymbol{x}, \boldsymbol{X})\right) \cdot\left(\boldsymbol{\sigma}_{j}^{0}(\boldsymbol{x}, \boldsymbol{Y})-\boldsymbol{\sigma}_{j}(\boldsymbol{x}, \boldsymbol{Y})\right) \cdot \boldsymbol{n} \mathrm{d} s
\end{aligned}
$$

where $\boldsymbol{X}=X_{1} \boldsymbol{e}_{1}+X_{2} \boldsymbol{e}_{2}$ and $\boldsymbol{Y}=Y_{1} \boldsymbol{e}_{1}+Y_{2} \boldsymbol{e}_{2}$. We now specialize this formula $\eta$ and $\frac{\partial \eta}{\partial x}$ small. As pointed out by Kirchner and $\mathrm{Ni}$ (1993), the first two terms in (A.2) are of order 1 in $\eta$, while the last one is of order 2. Therefore, at the first order we can write:

$$
\left(\boldsymbol{N}_{j}(\boldsymbol{X}, \boldsymbol{Y})-\boldsymbol{N}_{j}^{0}(\boldsymbol{X}, \boldsymbol{Y})\right) \cdot \boldsymbol{e}_{i}=\int_{\Omega^{0}(0)-\Omega^{0}(\eta)} \boldsymbol{\epsilon}\left(\boldsymbol{N}_{j}^{0}\right)(\boldsymbol{x}, \boldsymbol{Y}): \boldsymbol{L}: \boldsymbol{\epsilon}\left(\boldsymbol{N}_{i}^{0}\right)(\boldsymbol{x}, \boldsymbol{X}) \mathrm{d} S
$$

This equation allows to determine the first order development of $\boldsymbol{N}_{j}(\boldsymbol{X}, \boldsymbol{Y})$ provided $\boldsymbol{N}_{j}^{0}(\boldsymbol{X}, \boldsymbol{Y})$ is known. Note that (A.3) can be rewritten as

$$
\left(\boldsymbol{N}_{j}(\boldsymbol{X}, \boldsymbol{Y})-\boldsymbol{N}_{j}^{0}(\boldsymbol{X}, \boldsymbol{Y})\right) \cdot \boldsymbol{e}_{i}=\int_{\Gamma^{0}(0)}\left[\boldsymbol{\epsilon}\left(\boldsymbol{N}_{j}^{0}\right)(x, \boldsymbol{Y}): \boldsymbol{L}: \boldsymbol{\epsilon}\left(\boldsymbol{N}_{i}^{0}\right)(x, \boldsymbol{X})\right] \eta(x) \mathrm{d} x
$$

where $\boldsymbol{\epsilon}\left(\boldsymbol{N}_{j}^{0}\right)(x, \boldsymbol{Y})$ is the value of $\boldsymbol{\epsilon}\left(\boldsymbol{N}_{j}^{0}\right)(\boldsymbol{x}, \boldsymbol{Y})$ at the point $\boldsymbol{x}=x \boldsymbol{e}_{1}+\eta(x) \boldsymbol{e}_{2}$. 
When $\boldsymbol{X}=X_{1} \boldsymbol{e}_{1}+X_{2} \boldsymbol{e}_{2}$ and $\boldsymbol{Y}=Y_{1} \boldsymbol{e}_{1}+Y_{2} \boldsymbol{e}_{2}$ tend to the surface $\Gamma^{0}(\eta)$, then $\boldsymbol{N}_{j}(\boldsymbol{X}, \boldsymbol{Y}) \boldsymbol{e}_{i}$ tends to the function $N_{i j}\left(\eta, X_{1}, X_{2}\right)$ defined in (12). Moreover in that case, an expansion at the first order in $\eta$ enables to write $\boldsymbol{N}_{j}^{0}(\boldsymbol{X}, \boldsymbol{Y})$ as a function of its values $N_{i j}^{0}\left(X_{0}, Y_{0}\right)=\boldsymbol{e}_{i} \cdot \boldsymbol{N}_{j}^{0}\left(X_{0} \boldsymbol{e}_{1}, Y_{0} \boldsymbol{e}_{1}\right)$ on the surface $\Gamma^{0}(0)$. Substituting in (A.4) provides

$$
\begin{aligned}
& N_{i j}\left(\eta, X_{1}, Y_{1}\right)-N_{i j}^{0}\left(X_{1}, Y_{1}\right) \\
& \quad=\eta\left(X_{1}\right) \frac{\partial \boldsymbol{N}_{j}^{0}}{\partial n_{X}}\left(X_{1}, Y_{1}\right) \cdot \boldsymbol{e}_{i}+\eta\left(Y_{1}\right) \frac{\partial \boldsymbol{N}_{j}^{0}}{\partial n_{Y}}\left(X_{1}, Y_{1}\right) \cdot \boldsymbol{e}_{i}+\int_{\Gamma^{0}(0)}\left[\boldsymbol{\epsilon}\left(\boldsymbol{N}_{j}^{0}\right)\left(x, Y_{1}\right): \boldsymbol{L}: \boldsymbol{\epsilon}\left(\boldsymbol{N}_{i}^{0}\right)\left(x, X_{1}\right)\right] \eta(x) \mathrm{d} x
\end{aligned}
$$

with

$$
\begin{aligned}
& \frac{\partial \boldsymbol{N}_{j}^{0}}{\partial n_{X}}\left(X_{1}, Y_{1}\right)=\lim _{\delta \rightarrow 0} \frac{\boldsymbol{N}_{j}^{0}\left(X_{1} \boldsymbol{e}_{1}+\delta \boldsymbol{e}_{2}, Y_{1} \boldsymbol{e}_{1}\right)-\boldsymbol{N}_{j}^{0}\left(X_{1} \boldsymbol{e}_{1}, Y_{1} \boldsymbol{e}_{1}\right)}{\delta} \\
& \frac{\partial \boldsymbol{N}_{j}^{0}}{\partial n_{Y}}\left(X_{1}, Y_{1}\right)=\lim _{\delta \rightarrow 0} \frac{\boldsymbol{N}_{j}^{0}\left(X_{1} \boldsymbol{e}_{1}, Y_{1} \boldsymbol{e}_{1}+\delta \boldsymbol{e}_{2}\right)-\boldsymbol{N}_{j}^{0}\left(X_{1} \boldsymbol{e}_{1}, Y_{1} \boldsymbol{e}_{1}\right)}{\delta}
\end{aligned}
$$

and $\boldsymbol{\epsilon}\left(\boldsymbol{N}_{j}^{0}\right)\left(x, Y_{1}\right)$ is the value of $\boldsymbol{\epsilon}\left(\boldsymbol{N}_{j}^{0}\right)(x, \boldsymbol{Y})$ at the point $\boldsymbol{Y}=Y_{1} \boldsymbol{e}_{1}+\eta\left(Y_{1}\right) \boldsymbol{e}_{2}$.

Le $\boldsymbol{u}$ be the displacement solution of the problem (11). On the surface we have

$$
u_{i}(x)=\sum_{j=1}^{2} \int_{-b}^{b} N_{i j}\left(\eta, x, x^{\prime}\right) T_{j}\left(x^{\prime}\right) \mathrm{d} x^{\prime}
$$

where $T_{j}\left(x^{\prime}\right)$ is supposed to be null outside the segment $[-b, b]$. Substituting (A.5) in (A.8) gives

$$
\begin{aligned}
u_{i}(x)= & \sum_{j=1}^{2} \int_{-b}^{b} N_{i j}^{0}\left(x, x^{\prime}\right) T_{j}\left(x^{\prime}\right) \mathrm{d} x^{\prime}+\eta(x) \int_{-b}^{b} T_{j}\left(x^{\prime}\right) \frac{\partial \boldsymbol{N}_{j}^{0}}{\partial n_{X}}\left(x, x^{\prime}\right) \cdot \boldsymbol{e}_{i} \mathrm{~d} x^{\prime}+\int_{-b}^{b} T_{j}\left(x^{\prime}\right) \eta\left(x^{\prime}\right) \frac{\partial \boldsymbol{N}_{j}^{0}}{\partial n_{Y}}\left(x, x^{\prime}\right) \cdot \boldsymbol{e}_{i} \mathrm{~d} x^{\prime} \\
& +\int_{-b}^{b} \int_{-b}^{b}\left[\boldsymbol{\epsilon}\left(\boldsymbol{N}_{j}^{0}\right)\left(z, x^{\prime}\right): \boldsymbol{L}: \boldsymbol{\epsilon}\left(\boldsymbol{N}_{i}^{0}\right)(z, x)\right] T_{j}\left(x^{\prime}\right) \eta(z) \mathrm{d} z \mathrm{~d} x^{\prime}
\end{aligned}
$$

Since $N^{0}$ is known, one can go through the calculation of (A.9) to obtain the expression of $u_{i}(x)$. The result is given by Eq. (15).

\section{Appendix B. Existence of solutions for the discretized problem}

The existence of solutions to the optimization problem (33) is now proved. With the regularization (20) of the contact conditions, the contact tractions depend continuously on $\boldsymbol{p}$. Therefore, $\boldsymbol{G}_{i}(\boldsymbol{p})$ is a continuous function of $\boldsymbol{p}$, which implies that the set $F_{1}=\left\{\boldsymbol{p}, \boldsymbol{G}_{i}(\boldsymbol{p}) \leqslant G_{c} \boldsymbol{I}\right.$ for $\left.1 \leqslant i \leqslant m\right\}$ is closed. Consequently the set $F_{2}$ defined by $F_{2}=\{\boldsymbol{p}, \boldsymbol{p} \geqslant 0\} \cap F_{1}$ is closed as well. Note that $F_{2}$ is not empty because for $\boldsymbol{p} \geqslant 0$ such that $|\boldsymbol{p}|$ is sufficiently large, $\boldsymbol{G}_{i}(\boldsymbol{p})$ is equal to zero (there is no contact). Since the function $J^{h}$ becomes infinite as $|\boldsymbol{p}|$ grows to infinity, it has some minima on the closed nonempty set $F_{2}$. Hence the existence of solutions to (32) is ensured. The same result can be proved for (33) using the fact that $J_{\alpha}^{h}$ becomes infinite as $|\boldsymbol{p}|$ grows to infinity.

It can also be proved that the solutions of (33) converge towards the solutions of (32) when the parameter $\alpha$ tend to 0 . Let $\lambda$ be a solution of (32) and $\left\{\alpha_{n}\right\}$ a positive sequence converging towards 0 . For each $n$, we denote by $\boldsymbol{p}_{n}$ a solution of (33). The following inequalities hold:

$$
\left|\boldsymbol{p}_{n}\right| \leqslant J_{\alpha_{n}}^{h}\left(\boldsymbol{p}_{n}\right) \leqslant J_{\alpha_{n}}^{h}(\boldsymbol{\lambda})=|\lambda|
$$


Hence the sequence $\left\{\boldsymbol{p}_{n}\right\}$ is bounded. Consequently there exists a convergent subsequence, still denoted $\left\{\boldsymbol{p}_{n}\right\}$. Its limit $\boldsymbol{p}_{\infty}$ necessarily belongs to the closed set $\{\boldsymbol{p}, \boldsymbol{p} \geqslant 0\}$. For each $n$, we have:

$$
\frac{1}{\alpha_{n}} \sum_{i=1}^{m}\left\langle\boldsymbol{G}_{i}\left(\boldsymbol{p}_{n}\right)-G_{c} \boldsymbol{I}\right\rangle_{+} \cdot \Delta_{1} \cdot\left\langle\boldsymbol{G}_{i}\left(\boldsymbol{p}_{n}\right)-G_{c} \boldsymbol{I}\right\rangle_{+} \leqslant J_{\alpha_{n}}^{h}\left(\boldsymbol{p}_{n}\right) \leqslant|\lambda|
$$

whence

$$
\sum_{i=1}^{m}\left\langle\boldsymbol{G}_{i}\left(\boldsymbol{p}_{n}\right)-G_{c} \boldsymbol{I}\right\rangle_{+} \cdot \Delta_{1} \cdot\left\langle\boldsymbol{G}_{i}\left(\boldsymbol{p}_{n}\right)-G_{c} \boldsymbol{I}\right\rangle_{+} \leqslant \alpha_{n}|\lambda|
$$

Taking the limit $n \rightarrow \infty$ gives $\left\langle\boldsymbol{G}_{i}\left(\boldsymbol{p}_{\infty}\right)-G_{c} \boldsymbol{I}\right\rangle_{+}=0$ i.e., $\boldsymbol{p}_{\infty} \in F_{1}$. Since $\boldsymbol{p}_{\infty} \geqslant 0$, we also have $\boldsymbol{p}_{\infty} \in F_{2}$. The inequality (B.1) shows that $\left|\boldsymbol{p}_{\infty}\right| \leqslant|\boldsymbol{\lambda}|$, so $\boldsymbol{p}_{\infty}$ is solution of (32).

\section{References}

Barquin, M., Maugis, D., Courtel, R., 1974. Figure d'équilibre d'une surface de caoutchouc sous l'action de frottement d'une bille de verre. C. R. Acad. Sci. Paris 279, 565-569.

Bui, H.D., Dang Van, K., 1976. Contribution à l'étude théorique du contact élastique d'un frotteur cylindrique glissant sur un massif élastique. Colloque annuel du Groupe français de, 1-10.

Bui, H.D., 1994. Inverse Problems in the Mechanics of Materials: An Introduction. CRC Press.

Dragon-Louiset, M., 2001. On a predictive macroscopic contact-sliding wear model based on micro-mechanical considerations. Int. J. Solid Struct. 38, 1625-1639.

Fletcher, R., 1987. Practical Methods of Optimization. John Wiley \& Sons.

Fouvry, S., 2001. Shakedown analysis and fretting wear response under gross slip condition. Wear 251, 1320-1331.

Halphen, B., Nguyen, Q.S., 1975. Sur les matériaux standards généralisés. J. Mécanique 14, 39-63.

Johnson, K.L., 1985. Contact Mechanics. Cambridge University.

Kirchner, H.O.K., Ni, L., 1993. Domain dependence of Elastic Green's, Hadamard's and Bergmann's functions. J. Mech. Phys. Solids 41, 1461-1478.

Koiter, W.T., 1960. General theorems for elastic-plastic solids. Prog. Solid Mech. 1, 165-221.

Meng, H.C., Ludema, K.C., 1995. Wear models and predictive equations - their form and content. Wear 181, $443-457$.

Oden, J.T., Martins, J.A.C., 1985. Models and computational methods for dynamic friction phenomena. Comput. Meth. Appl. Mech. Eng. 52, 527-634.

Öqvist, M., 2001. Numerical simulations of mild wear using updated geometry with different step sizes approaches. Wear 49, 6-11.

Peigney, M., Stolz, C., 2003. An optimal control approach to the analysis of inelastic structures under cyclic loading. J. Mech. Phys. Solids 51 (4), 575-605.

Põdra, P., Andersonn, S., 1999. Simulating sliding wear with finite element method. Tribol. Int. 32, 71-81.

Pradeilles-Duval, R.M., Stolz, C., 1995. Mechanical transformations and discontinuities along a moving surface. J. Mech. Phys. Solids 43 (1), 91-121.

Tiba, D., 1990. Optimal control of non-smooth distributed parameter systems. In: Lecture Notes in Mathematics, 1459. Springer Verlag. 